\title{
Replanteando el Bulevar: Un jardín vertical como intervención artística y comunitaria
}

\author{
Rethinking the Boulevard: A vertical garden as an artistic and \\ community intervention
}

Mar CASTILLEJO. Centro Municipal de Salud Comunitaria de Puente de Vallecas (España).marcastillejohigueras@gmail.com

Resumen: El siguiente artículo presenta "Replanteando el bulevar", una iniciativa de intervención a modo de jardín vertical construido de manera colaborativa entre vecinas y vecinos del barrio, estudiantes del Máster de Educación Artística en Instituciones Sociales y Culturales de la Universidad Complutense de Madrid, y profesionales del Centro Municipal de Salud Comunitaria (en adelante CMSc) de Puente de Vallecas, para la fachada principal de este centro. El objetivo que se plantea es convertir el espacio colindante al CMSc, que se caracteriza por sus problemáticas diversas (limpieza, problemas de convivencia, estigmatización, etc.) en un punto de encuentro saludable a través de los cuidados y la participación. Dicha intervención se ha desarrollado en distintas fases (desde Noviembre de 2017 a Junio de 2018) en las que se configura un grupo promotor que junto con la comunidad va construyendo y repensando este espacio en diferentes eventos comunitarios desarrollados en el distrito. De este modo, se ha recurrido a metodologías propias del arte colaborativo para diseñar una propuesta creativa que permitiese alcanzar un objetivo relacionado con la promoción de la salud. Se puede concluir que el mantenimiento del jardín a través de los cuidados de vecinas, vecinos y profesionales del CMSc, evidencia que este tipo de iniciativas pueden resultar útiles para implicar a la comunidad en la creación y replanteamiento de espacios tradicionalmente estigmatizados en lugares más saludables.

Palabras clave: prácticas artísticas colaborativas, salud comunitaria, entorno, participación, cuidados, jardinería vecinal. 


\begin{abstract}
The following article presents "Rethinking the boulevard", an intervention initiative as a vertical garden built in a collaborative way between residents of the neighbourhood, students of the Master's Degree in Artistic Education in Social and Cultural Institutions of the Complutense University of Madrid, and professionals from the Municipal Centre for Community Health (CMSc) of Puente de Vallecas, for the main facade of this centre. The objective is to turn the space adjacent to the CMSc, which is characterized by its diverse problems (cleanliness, problems of coexistence, stigmatization, etc.) into a healthy meeting point through care and participation. This intervention has been developed in different phases (from November 2017 to June 2018) in which a promoting group is formed that together with the community is building and rethinking this space through activities carried out in community events. In this way, we have resorted to collaborative art methodologies to design a creative proposal that would allow us to achieve an objective related to health promotion. It can be concluded that the maintenance of the garden through the care of neighbors and professionals of the CMSc, shows that this type of initiative can be useful to involve the community in the creation and rethinking of spaces in healthier places.
\end{abstract}

Keywords: collaborative artistic practices, community health, environment, participation, care, neighborhood gardening.

\title{
Introducción
}

Este artículo describe una experiencia concreta que se enmarca dentro de lo que se define como community-based arts (proyectos comunitarios basados en el arte) (Cleveland, 2000). En ellos, se parte de la premisa de que "el arte puede ser una actividad vinculada a la comunidad, al contexto específico y plantea la posibilidad de colaborar en la construcción de una alternativa colectiva que participe activamente en los procesos de transformación social" (Parramón 2003), Así mismo, refuerza la idea de que este tipo de proyectos permiten establecer colaboraciones entre diferentes campos de trabajo, en este caso entre el arte y la salud, los eventos participativos en el espacio público y la jardinería vecinal.

Resulta pertinente comenzar con una breve revisión acerca del origen y la evolución de este tipo de prácticas artísticas comunitarias. Según Alfredo Palacios (2009, p.199) se sitúa en torno a los años 70 en EEUU y Gran Bretaña. Se establece una relación entre "la evolución de las prácticas conceptuales, performativas y del arte de acción hacia el activismo sociopolítico", con las prácticas artísticas feministas y con un tipo de arte público cuyos objetivos subyacentes tienen que ver con "mejorar los espacios públicos donde vivía la clase trabajadora, promoviendo que fueran los propios residentes, agentes activos en la transformación de ese entorno", lo que (nos) permite comprender su posterior evolución hacia ámbitos institucionales y educativos también en el contexto español. 
En este marco, es inapelable poner además en relación este tipo de prácticas artísticas con la acción social, como destacan Rey, Delgado, Fernández-Cedena, y Sainz (2017) señalando que "las investigaciones del Observatorio del Tercer Sector de Bizkaia insisten en que el arte se convertiría en el instrumento para la participación y la transformación social en situaciones de exclusión, siendo las personas vulnerables las protagonista de los procesos de creación." y apuntando "una de las características esenciales de cualquier práctica artística colaborativa: más allá del valor estético de los productos, se hace especial hincapié en el proceso y la colaboración necesaria para la obtención de resultados" (Rey et al. 2017, p. 123). Fernández-Cedena (2017) vuelve a citar este mismo informe Arte para la inclusión y la transformación social (Sostegno y López-Arostegi 2012), en este caso para destacar que a menudo estas experiencias están ligadas a territorios que es necesario transformar.

En esta misma línea, Moreno afirma que:

El arte es una herramienta de primer orden para el trabajo con personas en situación de exclusión social para la inclusión y para el desarrollo comunitario, ya que a nivel individual promueve los procesos de simbolización, la resiliencia y el empoderamiento, a nivel grupal desarrolla cohesión y progreso; y a nivel comunitario favorece el desarrollo hacia sociedades inclusivas y más justas, que viven en paz acogiendo las diferencias. (Moreno, 2016, p.148)

Enmarcada en estas premisas, surge la iniciativa que aborda el presente artículo y que forma parte del proyecto de investigación "Arte y Salud". Dicho proyecto nace en 2012 con un acuerdo de colaboración entre el Organismo Autónomo Madrid Salud perteneciente al Ayuntamiento de Madrid y la facultad de Bellas Artes de la Universidad Complutense de Madrid (Proyecto Arte y Salud, 2018) El proyecto se fundamenta en el concepto compartido de proyecto comunitario, dando lugar a diferentes experiencias que validan estas iniciativas (Ávila, et al.2018).

Además, la posibilidad de trabajar en el contexto concreto que a continuación se describe, deriva de la incorporación en el marco de este proyecto, a través de becas de formación e investigación, de artistas a los equipos de los Centros Municipales de Salud Comunitaria en el año 2017, experiencia descrita por Castillejo, FernándezCedena, Siles, Claver y Ávila (2018).

El CMSc de Puente de Vallecas trabaja, llevando a la práctica la estrategia municipal "Barrios saludables" de la Subdirección general de Promoción y Prevención de la Salud (2019), la promoción de la salud de las vecinas y vecinos de uno de los distritos más densamente poblados de Madrid. El distrito de Puente de Vallecas, además presenta una serie de indicadores sociodemográficos que evidencian que nos encontramos en uno de los distritos de la ciudad de Madrid con mayor vulnerabilidad socio-económica: Alto nivel de desempleo, una de las cifras más altas de Madrid de perceptores de Renta Mínima de Inserción, etc. Por otro lado, 
las vecinas y vecinos del distrito conforman un tejido asociativo y presentan una identidad de barrio tradicionalmente reseñable.

El CMSc se sitúa en el Bulevar de Peña Gorbea, que ha sido durante décadas un lugar de encuentro para las vecinas y vecinos del barrio y un punto neurálgico de recursos Municipales: Biblioteca Municipal de Puente de Vallecas, Centro de Mayores La Casa Del Bulevar, Centro de Atención a las Adicciones; y comercios. Sin embargo, este espacio presenta diferentes problemáticas (carencia de limpieza, conflictos entre diferentes grupos de población, presencia de prostitución, problemas con vehículos mal estacionados que invaden el espacio público, estigmatización, situaciones personales de permanencia y consumo de tóxicos en este espacio, etc.) que impiden que la comunidad pueda disfrutar plenamente en este lugar.

Sobre esta base, el equipo del CMSc pone en marcha en junio de 2017 el "Proyecto Bulevar" para intentar convertirlo en un lugar más saludable desde una perspectiva amplia. Desde entonces se han ido programando actividades comunitarias en colaboración con diferentes entidades del distrito en este espacio.

La fachada del edificio es además un punto clave que urge intervenir, al ser el primer elemento que observan los vecinos y vecinas, entrando en contradicción su aspecto y condiciones de insalubridad (mal olor porque la gente orina en ella, cercanía de cubos de basura, etc.) con la labor que se desarrolla en un centro que se dedica a promocionar la salud en la comunidad.

"Replanteando el Bulevar" es una propuesta más, para pensar en conjunto cómo queremos que sea el barrio y más concretamente el bulevar, y qué necesitamos para conseguirlo. En pro de este objetivo, se recurre a prácticas participativas que utilizan el arte, la creatividad y el juego en el espacio público a partir de distintos eventos comunitarios en los que se van elaborando las diferentes piezas de lo que será la composición final de esta intervención.

Sobre la articulación de este tipo prácticas con algunos aspectos de la Atención Primaria en Salud desde una perspectiva Integral con enfoque en Salud Mental, ha escrito ampliamente la autora Claudia Bang. A través del estudio de caso de una red barrial de la ciudad argentina de Buenos Aires que desarrolla eventos comunitarios de este tipo, concluye que "estos eventos participativos de creación colectiva que utilizan el arte y el juego en el espacio público, en congruencia con las acciones de promoción de salud mental comunitaria, propician una vía hacia la transformación de los lazos comunitarios hacía vínculos solidarios y la participación comunitaria hacia la constitución de la propia comunidad como sujeto activo de transformación de sus realidades" (Bang, 2011).

En el caso de la intervención que nosotros describimos, se añaden además la perspectiva del cuidado y del mantenimiento extendido en el tiempo a través de los 
cuidados que implica una intervención realizada con elementos vegetales basada en la jardinería vecinal. En este sentido contamos con referentes similares llevados a cabo en otros barrios de Madrid, tales como Tu barrio se planta.

\section{Objetivos}

Objetivo general

- Transformar la fachada del CMSc en un punto saludable del bulevar para la participación de las vecinas y vecinos del distrito.

Objetivos específicos

- Generar vínculos entre vecinas, vecinos y entidades a través de dinámicas que tienen que ver con los cuidados.

- Dar visibilidad al CMSc de Puente de Vallecas.

\section{Metodología}

Como técnica metodológica se ha recurrido fundamentalmente a la Investigación acción participativa. El diseño de la intervención parte del sentir general de la comunidad de que es necesario proponer alternativas para mejorar el estado del bulevar, así como del diagnóstico realizado por profesionales del CMSc, según el cual es apremiante mejorar el entorno en el que trabajan en promoción de la salud. Esta percepción colectiva se hace patente en diferentes actividades de mapeo desarrolladas en el barrio de San Diego, donde las alusiones a este espacio son numerosas y se relacionan mayoritariamente con problemáticas de limpieza y con conflictos entre diferentes grupos de población. Diferentes comentarios extraídos de uno de los mapas emocionales realizados, ejemplifican y relacionan el bulevar con un sentimiento de tristeza: "Suciedad, pobreza, presencia de alcohólicos, pisos okupas, echado a perder" o " Suciedad y deterioro; escaso cuidado de la zona por parte de los vecinos; presencia de personas con problemas de alcoholismo y drogadicción; gente durmiendo en la calle; sensación de inseguridad; ahora es menos acogedor que antes para los niños; lo vacías que están las casetas del bulevar".

Estas referencias a diferentes mapas emocionales, sumados al análisis de documentos previos en torno a estas cuestiones, tales como el "Plan de acciones del prediagnóstico de Puente de Vallecas" (Paisaje transversal y todo por la praxis, 2017), han permitido componer un diagnóstico inicial. Sobre la base del mismo, ha sido posible elaborar una propuesta que responda en la medida de sus posibilidades a las necesidades de la comunidad, desarrollando en paralelo una investigación, que pretende ser como proponen Ballesteros, Del Olmo y Mata (2014), socialmente útil.

Por otra parte, para conseguir los objetivos anteriormente enunciados resulta fundamental contar con la implicación de diferentes agentes que comparten este 
espacio. En este sentido, la red informal que se ha ido generando entre entidades y colectivos a través de la propia realización de eventos comunitarios en el bulevar, va a resultar una pieza clave para el desarrollo de esta experiencia.

Como ya anunciamos previamente, además de trabajar a través del potencial transformador para la comunidad de estos eventos en el espacio público, la necesidad de incorporar los cuidados a largo plazo dentro del proyecto, nos lleva a plantear la cuestión de la intervención a base de vegetales, que necesitan un mantenimiento posterior. Esta elección nos permite además evaluar algunos aspectos de la misma.

De igual manera, la posibilidad de contar con un grupo de estudiantes del Máster de Educación Artística en Instituciones Sociales y Culturales de la Universidad Complutense de Madrid, se plantea como una oportunidad para trabajar a través de la hibridación de saberes múltiples, sinergias entre arte, salud y comunidad.

A continuación, se describen las diferentes fases en las que este proceso se ha desarrollado y las actividades puestas en marcha.

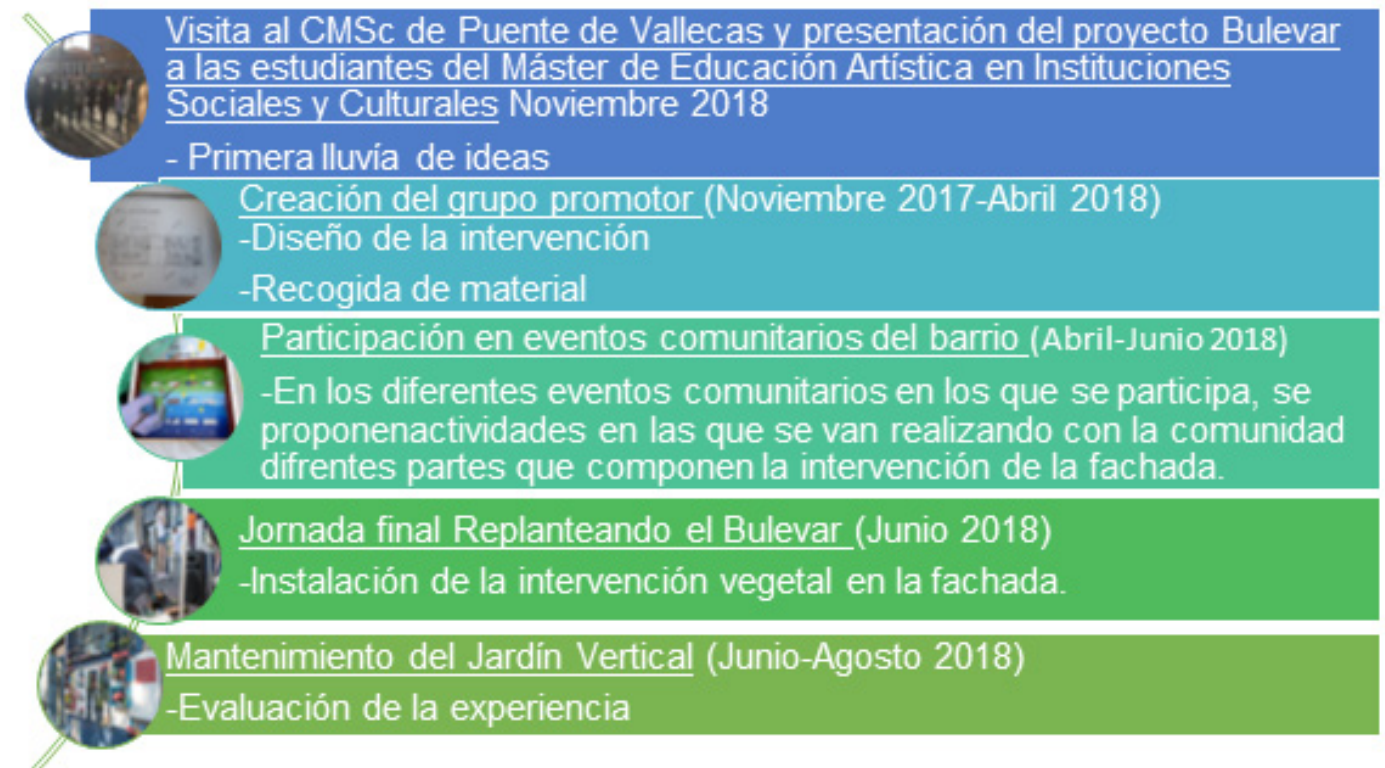

Figura 1. Fases de la intervención

En la tabla 1 se muestran pormenorizadamente las propuestas realizadas en los diferentes eventos comunitarios del distrito que incluyen actividades inscritas en el proyecto. En primer lugar se señala el evento y se describe brevemente en qué consiste, para después describir la actividad concreta que el grupo promotor propone. Se registra también la participación en esta actividad y se señalan aspectos que pueden resultar interesantes para responder a los objetivos del proyecto. Las tres primeras columnas recogen aspectos metodológicos y las dos últimas datos extraídos. 
Tabla 1

Aspectos metodológicos y datos extraídos.

\begin{tabular}{|c|c|c|c|c|}
\hline $\begin{array}{l}\text { Evento } \\
\text { Comunitario }\end{array}$ & $\begin{array}{l}\text { Descripción del } \\
\text { evento }\end{array}$ & $\begin{array}{l}\text { Descripción } \\
\text { de la actividad } \\
\text { propuesta } \\
\text { por el GP de } \\
\text { Replanteando el } \\
\text { Bluevar }\end{array}$ & $\begin{array}{l}\text { Participantes en } \\
\text { el taller }\end{array}$ & Datos relevantes \\
\hline $\begin{array}{l}\text { Historias en el } \\
\text { camino }\end{array}$ & $\begin{array}{l}\text { Actividad } \\
\text { comunitaria para } \\
\text { celebrar el Día } \\
\text { del Libro en el } \\
\text { Bulevar de Peña } \\
\text { Gorbea. }\end{array}$ & $\begin{array}{l}\text { Taller de } \\
\text { decoración de } \\
\text { cajas de fruta y } \\
\text { palets. }\end{array}$ & 27 niñas y niños & $\begin{array}{l}\text { Familias con } \\
\text { niñas y niños } \\
\text { pequeños que } \\
\text { nunca acuden } \\
\text { a esta parte } \\
\text { del bulevar } \\
\text { debido a la } \\
\text { estigmatización } \\
\text { de las } \\
\text { personas que } \\
\text { habitualmente } \\
\text { habitan en él, } \\
\text { comparten } \\
\text { espacio con } \\
\text { ellas y ellos, y } \\
\text { colaboran en la } \\
\text { actividad. }\end{array}$ \\
\hline $\begin{array}{l}\text { Día del Libro: } \\
\text { Trueque de } \\
\text { libros, cuenta } \\
\text { cuentos y talleres } \\
\text { infantiles en el } \\
\text { Pozo del Tío } \\
\text { Raimundo }\end{array}$ & $\begin{array}{l}\text { Actividad } \\
\text { comunitaria en } \\
\text { el Pozo del Tío } \\
\text { Raimundo. }\end{array}$ & $\begin{array}{l}\text { Taller de } \\
\text { decoración de } \\
\text { cajas de frutas. }\end{array}$ & 15 adolescentes & $\begin{array}{l}\text { Más organización } \\
\text { que en la } \\
\text { anterior. Mejores } \\
\text { resultados } \\
\text { artísticos. } \\
\text { Se establece } \\
\text { contacto con } \\
\text { la Asociación } \\
\text { Vecinal } \\
\text { para futuras } \\
\text { colaboraciones. }\end{array}$ \\
\hline Vive el Bulevar & $\begin{array}{l}\text { Actividad } \\
\text { Comunitaria que } \\
\text { se realiza por } \\
\text { segundo año en } \\
\text { el Bulevar de } \\
\text { Peña Gorbea. }\end{array}$ & $\begin{array}{l}\text { Taller de macetas } \\
\text { con botellas. }\end{array}$ & $\begin{array}{l}12 \text { Participantes. } \\
\text { Población } \\
\text { general. }\end{array}$ & $\begin{array}{l}\text { Participan al } \\
\text { mismo tiempo } \\
\text { vecinas y vecinos } \\
\text { y personas } \\
\text { estigmatizadas } \\
\text { dentro de este } \\
\text { espacio. }\end{array}$ \\
\hline $\begin{array}{l}\text { Día de la Infancia } \\
\text { en Vallecas }\end{array}$ & $\begin{array}{l}\text { Actividad } \\
\text { comunitaria } \\
\text { que se realiza } \\
\text { anualmente para } \\
\text { celebrar el día de } \\
\text { la Infancia. }\end{array}$ & $\begin{array}{l}\text { Taller de macetas } \\
\text { con botellas. }\end{array}$ & 42 Niñas y niños & $\begin{array}{l}\text { Se da a conocer } \\
\text { el proyecto fuera } \\
\text { del espacio del } \\
\text { Bulevar. }\end{array}$ \\
\hline
\end{tabular}




\begin{tabular}{|c|c|c|c|c|}
\hline $\begin{array}{l}\text { Jornada } \\
\text { "Arte, Salud y } \\
\text { Cuidados" }\end{array}$ & $\begin{array}{l}\text { Jornada realizada } \\
\text { en Medialab } \\
\text { Prado para } \\
\text { dar a conocer } \\
\text { el proyecto } \\
\text { "Arte, Salud y } \\
\text { Cuidados". }\end{array}$ & $\begin{array}{l}\text { Taller Moviliza } \\
\text { tu barrio. } \\
\text { Replanteando } \\
\text { el Bulevar. } \\
\text { Se fabrican } \\
\text { semilleros y } \\
\text { marcadores para } \\
\text { plantas con la } \\
\text { pregunta ¿qué } \\
\text { te gustaria que } \\
\text { germinase en su } \\
\text { barrio? y ¿Qué } \\
\text { cuidados que } \\
\text { necesita para } \\
\text { crecer? }\end{array}$ & $\begin{array}{l}15 \text { Profesionales } \\
\text { de Madrid Salud } \\
\text { y otras entidades. }\end{array}$ & $\begin{array}{l}\text { Mensajes } \\
\text { positivos en los } \\
\text { marcadores de } \\
\text { plantas. }\end{array}$ \\
\hline \multirow[t]{2}{*}{$\begin{array}{l}\text { Jornada Final } \\
\text { "Replanteando el } \\
\text { Bulevar" }\end{array}$} & $\begin{array}{l}\text { Jornada de } \\
\text { montaje e } \\
\text { instalación de la } \\
\text { intervención en } \\
\text { la fachada. }\end{array}$ & $\begin{array}{l}\text { Jornada festiva } \\
\text { y lúdica en la } \\
\text { que se instalan } \\
\text { y se unen cada } \\
\text { una de las partes } \\
\text { fabricadas en } \\
\text { las actividades } \\
\text { anteriores y se } \\
\text { trasplantan los } \\
\text { semilleros. }\end{array}$ & $\begin{array}{l}39 \text { Población } \\
\text { general. } \\
\text { Destacamos la } \\
\text { participación } \\
\text { del colectivo } \\
\text { de personas en } \\
\text { situaciones de } \\
\text { estigmatización y } \\
\text { vulnerabilidad. }\end{array}$ & $\begin{array}{l}\text { Personas en } \\
\text { situaciones de } \\
\text { estigmatización } \\
\text { colaboran y se } \\
\text { implican en la } \\
\text { realización de } \\
\text { la intervención. } \\
\text { Se recogen } \\
\text { sugerencias e } \\
\text { ideas de mejoras } \\
\text { y cuidados } \\
\text { para el barrio } \\
\text { y los espacios } \\
\text { públicos. }\end{array}$ \\
\hline & & & & $\begin{array}{l}\text { En los } \\
\text { marcadores se } \\
\text { aprecia la fuerte } \\
\text { Identidad de } \\
\text { barrio de Vallecas } \\
\text { así como la } \\
\text { diversidad y } \\
\text { multiculturalidad } \\
\text { de sus vecinas y } \\
\text { vecinos. }\end{array}$ \\
\hline
\end{tabular}

En las siguiente figura podemos observar una nube de palabras que recoge las respuestas obtenidas en los marcadores de plantas realizados en las actividades para la creación de semilleros. 


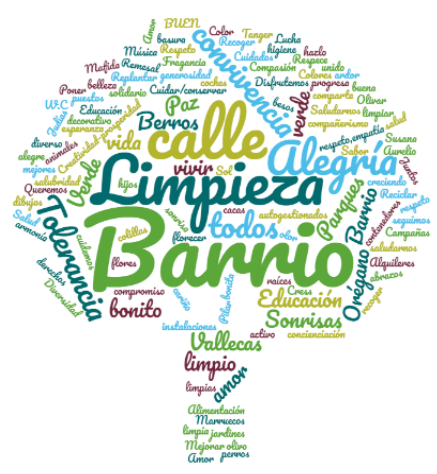

Figura 2. Nube de palabras

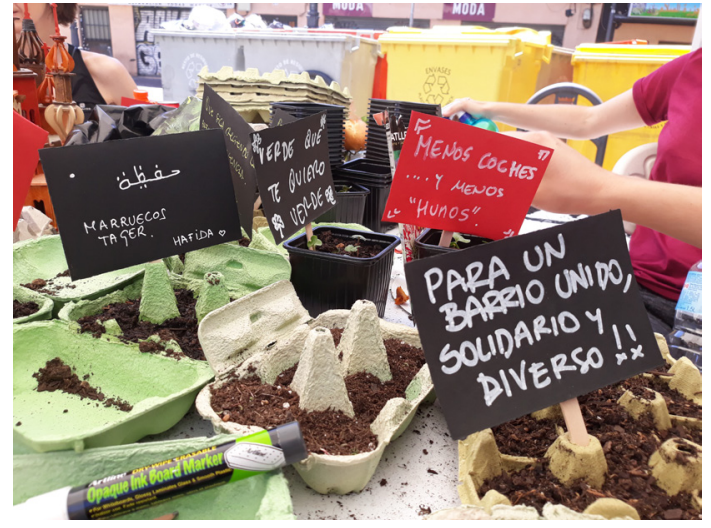

Figura 3. Semilleros

Además de las actividades anteriormente descritas, es necesario señalar que durante el proceso, diferentes grupos han colaborado de manera informal en el cuidado de los semilleros hasta que estos fueron trasplantados (diferentes usuarios del CMSc y un grupo de personas mayores del programa de envejecimiento activo y saludable) y en la recogida de material reciclable para las distintas actividades (grupo de personas en situación de estigmatización, vulnerabilidad y de calle, que normalmente habitan el espacio del bulevar en el que se realiza la intervención).

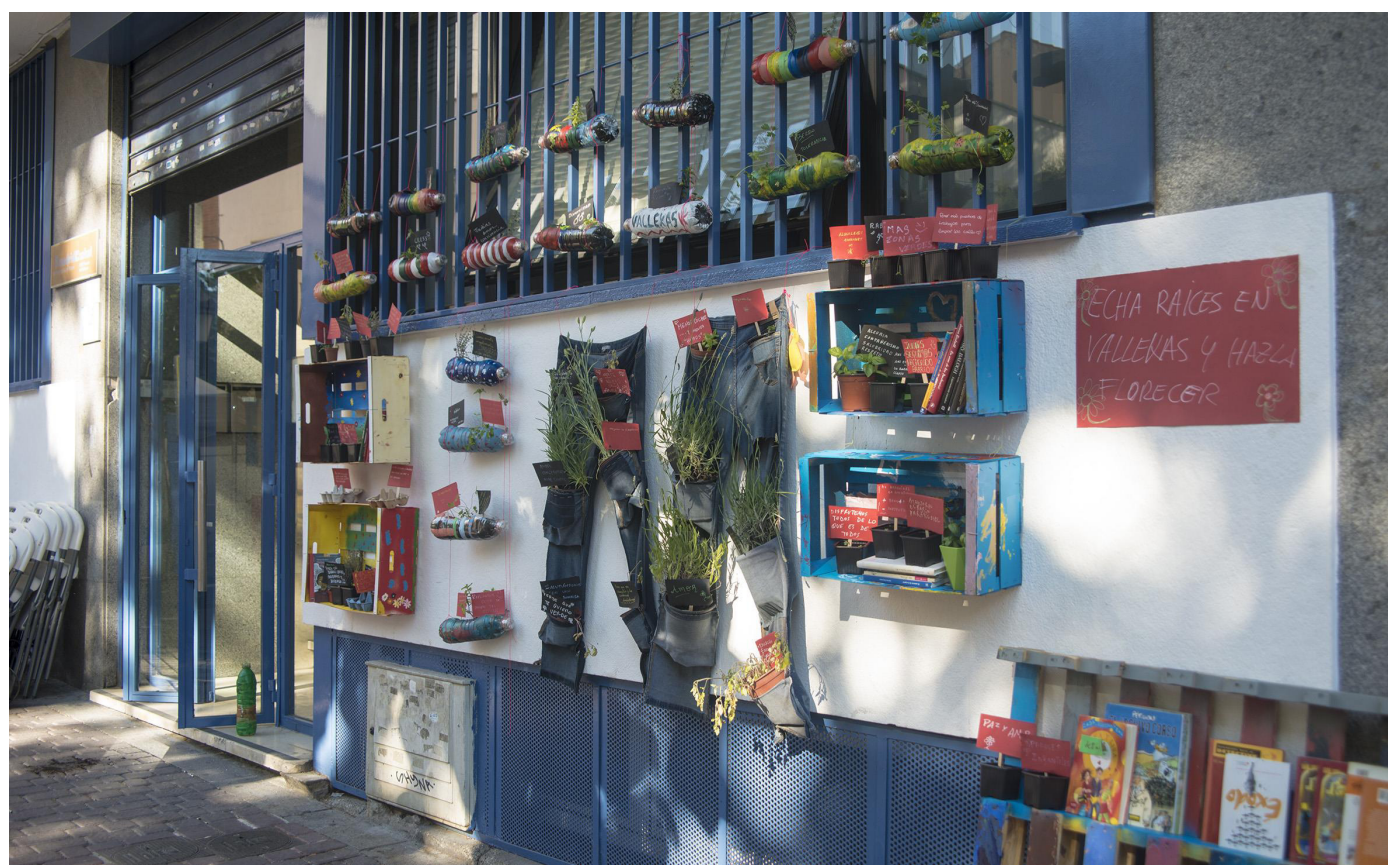

Figura 4. Fotografía de la intervención en la fachada.

De igual modo, se destaca especialmente la importancia de la última fase del proceso, que tiene que ver con el mantenimiento de la instalación, y que además posibilita desarrollar una evaluación creativa de la experiencia. 


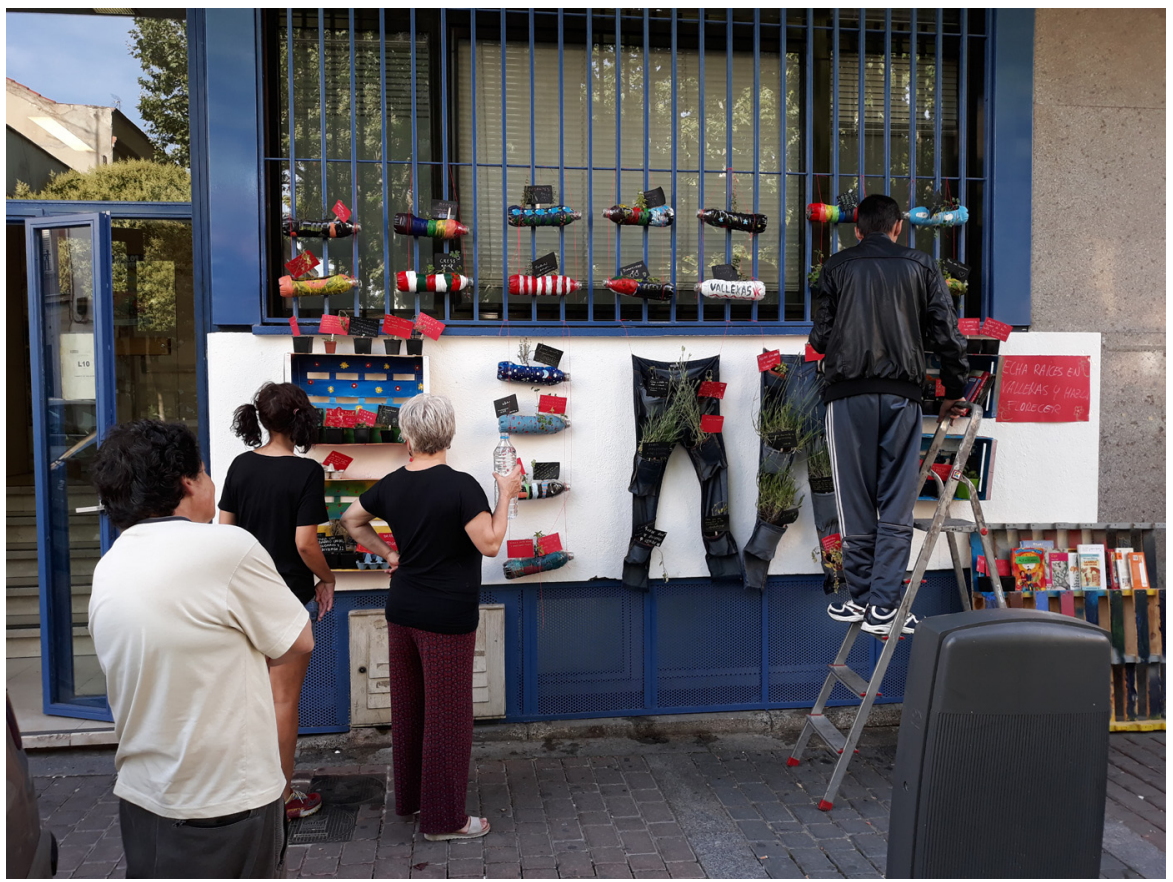

Figura 5. Vecinos y profesionales regando el jardín.

\section{Conclusiones}

Considerando los resultados señalados en las dos últimas columnas de la tabla 2 y algunos datos cualitativos obtenidos de la observación participante de esta experiencia, a continuación, se exponen algunas conclusiones elaboradas a partir del desarrollo de las distintas fases del proyecto.

Conviene destacar que en procesos complejos como el que se ha desarrollado, resulta difícil señalar las transformaciones concretas que se hayan producido correlativamente a la realización del mismo. Este hecho se explica porque los cambios que se pueden observar en una comunidad son siempre multifactoriales y a largo plazo. Sin embargo, es importante señalar que como consecuencia de este tipo de proyectos se pueden generar nuevos imaginarios colectivos que posibilitan una redefinición de espacios tradicionalmente denostados.

Partiendo de la observación participante como técnica empleada en las diferentes fases del proyecto, y prestando especial atención a los eventos comunitarios en los que se han llevado a cabo las diferentes actividades enunciadas anteriormente, podemos describir cuestiones que tienen que ver con los siguientes cambios:

En primer lugar resultan especialmente significativas las posibilidades de legitimar la presencia de personas normalmente estigmatizadas, como el grupo de personas en distintas situaciones de vulnerabilidad o de calle que normalmente habitan este espacio. Gracias a procesos de este tipo, en los que se sienten invitados a participar y en los cuales aprecian que lo que hacen tiene valor, y que además se relacionan 
directamente con un sentimiento positivo que permite "salir de la rutina, dar un toque de alegría" según las palabras recogidas de uno de los participantes. Así mismo, la oportunidad de encontrarse con otras vecinas y vecinos del barrio en circunstancias diferentes a las habituales y colaborando en un proyecto común, resulta muy beneficioso como manera de proponer alternativas más saludables de convivencia.

Otra cuestión que conviene destacar, es la capacidad de establecer redes de colaboración en base a este tipo de prácticas. En el caso que nos ocupa, este hecho se da en cada evento en el que participan diferentes entidades y colectivos del barrio con los que las relaciones son cada vez más estrechas.

Por otra parte, como se ha venido señalando desde el inicio del artículo, la cuestión del mantenimiento posterior de la instalación vegetal es un aspecto fundamental del proyecto, que además nos permite obtener algunas claves pertinentes a la hora de evaluar la experiencia. Sobre esta base, la permanencia del jardín se considera un logro reseñable, partiendo de su ubicación en un espacio en el que muchas vecinas y vecinos dudaban de su continuidad. Sin embargo, la instalación se mantuvo durante más de dos meses gracias a la implicación en su cuidado de vecinas y vecinos que bien habitan el bulevar o transitan a menudo por este espacio. En última instancia, la retirada final del mismo se debió al deterioro de los materiales empleados para su construcción. Por ende, se puede concluir que la incorporación de elementos que precisen mantenimiento, en este tipo de propuestas fomenta la implicación de diferentes agentes en el cuidado del entorno.

Igualmente destacable resulta el hecho de que el punto de intercambio de libros que se instaló complementando el jardín se mantuviese vivo durante estos dos meses. Aunque en ocasiones los objetos intercambiados no tuvieran nada que ver con la lectura (figuras decorativas, etc.). Estos hechos ponen de manifiesto que propuestas de este tipo generan espacios de participación para la comunidad.

\section{Discusión}

La transformación de la fachada del CMSc de Puente de Vallecas a través de una intervención vegetal y un punto de intercambio de libros construidos con la comunidad, ha servido para:

- Generar nuevos vínculos con vecinas y vecinos del barrio.

- Continuar desarrollando sinergias con otras entidades a través de la participación en eventos comunitarios.

- Proporcionar la posibilidad a las personas en situaciones de estigmatización 
que habitan el bulevar de encontrarse en su barrio de otras maneras.

- Dar visibilidad al CMSc y a la labor de este en el distrito.

Dando así un pequeño paso que contribuye a la transformación de este lugar en un espacio más saludable.

En base a lo anteriormente expuesto, es importante continuar trabajando para legitimar la validez de propuestas de este tipo, desarrollando investigaciones que visibilicen su potencial transformador e inviten a adaptar las propuestas en contextos similares.

\section{Referencias}

Avila N, Orellana A, Claver L, Segura J, Martínez M, Borrego O. (2018). Proyectos comunitarios: propuestas desde la promoción de la salud y la creación artística. Revista Comunidad. ISSN: 2339-7896. Recuperado de: http://comunidadsemfyc. es/proyectos/

Bang, C. (2011). Prácticas participativas que utilizan arte, creatividad y juego en el espacio público: Un estudio exploratorio desde la perspectiva de atención primaria de salud integral con enfoque en salud mental. Anuario de Investigaciones. Volumen 18. Facultad de Psicología-UBA. Secretaría de Investigaciones. ISSN 0329-5885. Recuperado de: http://repositoriouba.sisbi.uba.ar/gsdl/cgi-bin/library. cgi? $\mathrm{a}=\mathrm{d} \& \mathrm{c}=$ panuario $\& \mathrm{~d}=\mathrm{xviii}-71 \_\mathrm{htm}$

Ballesteros, B., Del Olmo, M., Mata, P. (2014) Propuestas de investigación e Intervención desde un enfoque participativo. Universidad Nacional de Educación a Distancia. ISBN:978-84-679-0623-7.

Castillejo, M., Fernández-Cedena, J., Siles, S., Ávila, N. y Claver, M. D. (2018) Batas Nómadas en Madrid Salud: el arte y los artistas en equipos profesionales de salud comunitaria. Gaceta Sanitaria, 32(5), 466-472. Recuperado de: http://www.gacetasanitaria.org/es-batas-nomadas-madrid-salud-elarticulo-S0213911118300955

Castillo, R., Sostegno, R., y López-Arostegi, R. (2012). Arte para la inclusión y la transformación social. Observatorio del Tercer Sector de Bizkaia. Recuperado de: http://www.3sbizkaia.org/Archivos/Documentos/Enlaces/1363_CASTinnovacion04.pdf

Cleveland W. (2000) Art in Other Places: Artists at Work in America's Community and Social Institutions. New York: Edita Praeger. 
Fernández-Cedena, J. (2017). Arte y acción social en prisiones. Diseñando un taller permanente, primeras experiencias. EARI. Educación Artística. Revista de Investigación, 8, 86-104. doi: 10.7203/eari.8.10020

Madrid Salud. Instituto de Salud Pública. Subdirección general de Prevención y Promoción. Barrios Saludables: La estrategia municipal de promoción de la salud 2016-2019. Recuperado de: https:/www.madrid.es/UnidadesDescentralizadas/ Salud/Prevencion YPromocion/EspInformativos/Estrategia \%20gente $\% 20$ saludable/ficheros/BARRIOS_SALUDABLES_2018.pdf

Moreno González, A. (2016). La mediación artística (1st ed.). Barcelona: Octaedro.

Paisaje transversal y Todo por la Praxis (2017). Plan de Acciones del Prediagnóstico de Puente de Vallecas.

Palacios, A. (2009). El arte comunitario: Origen y evolución de las prácticas artísticas colaborativas. Arteterapia-Papeles de arteterapia y educación artística para la inclusión social, 4, 197-2011. ISSN: 1886-6190.

Parramón, R. (Octubre, 2003). Arte, participación y espacio público. Models de participació en xarxa. Jornada d'innovació estratégica.

Rey, N., Delgado, L., Fernández-Cedena, J., y Sainz, S. (2017). Arte comunitario como herramienta de inclusión: experiencias en el Taller de Expresión Artística del Centro Penitenciario Madrid IV de Navalcarnero. EARI. Educación Artística. Revista de Investigación, 8, 120-141. doi: 10.7203/eari.8.9914

Universidad Complutense de Madrid (2018). Proyectos de Arte y Salud. Recuperado de: https:/www.ucm.es/arteysaludproyectos/ 